\title{
CONVERGENCE IN MEASURE OF STRONG LOGARITHMIC MEANS OF DOUBLE FOURIER SERIES
}

\author{
USHANGI GOGINAVA AND LARRY GOGOLADZE
}

\begin{abstract}
Nörlund strong logarithmic means of double Fourier series acting from space $L \log L\left(\mathbb{T}^{2}\right)$ into space $L_{p}\left(\mathbb{T}^{2}\right), 0<p<1$ are studied. The maximal Orlicz space such that the Nörlund strong logarithmic means of double Fourier series for the functions from this space converge in two-dimensional measure is found.
\end{abstract}

\section{INTRODUCTION}

The rectangular partial sums of double Fourier series $S_{n, m}(f ; x, y)$ of the function $f \in L_{p}\left(\mathbb{T}^{2}\right), \mathbb{T}:=[-\pi, \pi), 1<p<\infty$ converge in $L_{p}$ norm to the function $f$, as $n \rightarrow \infty$ 14. In the case $L_{1}\left(\mathbb{T}^{2}\right)$ this result does not hold . But for $f \in L_{1}(\mathbb{T})$, the operator $S_{n}(f ; x)$ are of weak type $(1,1)$ [16]. This estimate implies convergence of $S_{n}(f ; x)$ in measure on $\mathbb{T}$ to the function $f \in L_{1}(\mathbb{T})$. However, for double Fourier series this result does not hold [7, 9]. Moreover, it is proved that quadratical partial sums $S_{n, n}(f ; x, y)$ of double Fourier series do not converge in two-dimensional measure on $\mathbb{T}^{2}$ even for functions from Orlicz spaces wider than Orlicz space $L \log L\left(\mathbb{T}^{2}\right)$. On the other hand, it is well-known that if the function $f \in L \log L\left(\mathbb{T}^{2}\right)$, then rectangular partial sums $S_{n, m}(f ; x, y)$ converge in measure on $\mathbb{T}^{2}$.

Classical regular summation methods often improve the convergence of Fourier seeries. For instance, the Fejér means of the double Fourier series of the function $f \in L_{1}\left(\mathbb{T}^{2}\right)$ converge in $L_{1}\left(\mathbb{T}^{2}\right)$ norm to the function $f$ [14]. These means present the particular case of the Nörlund means.

The Nörlund logarithmic means of double Fourier series are defined by

$$
t_{n, m}(f ; x, y):=\frac{1}{l_{n} l_{m}} \sum_{i=0}^{n} \sum_{j=0}^{m} \frac{S_{i, j}(f ; x, y)}{(n-i+1)(m-j+1)},
$$

where $l_{n}:=\sum_{k=1}^{n+1}(1 / k)$ and by $S_{i, j}(f ; x, y)$ we denote rectangular partial sums of double Fourier series of the function $f$.

It is well know that the method of Nörlund logarithmic means of double Fourier series, is weaker than the Cesáro method of any positive order. In [10] Tkebuchava proved, that these means of double Fourier series in general do not converge in two-dimensional measure on $\mathbb{T}^{2}$ even for functions

\footnotetext{
${ }^{0} 2010$ Mathematics Subject Classification $42 \mathrm{~A} 24$.

Key words and phrases: double Fourier series, Orlicz space, Convergence in measure
} 
from Orlicz spaces wider than Orlicz space $L \log L\left(\mathbb{T}^{2}\right)$. For logarithmic means $t_{n, m}(f ; x, y)$ of double Fourier series Tkebuchava [11] proved that the following results are true.

Theorem 1. Let $L_{Q}\left(\mathbb{T}^{2}\right)$ be an Orlicz space, such that

$$
L_{Q}\left(\mathbb{T}^{2}\right) \nsubseteq L \log L\left(\mathbb{T}^{2}\right) \text {. }
$$

Then the set of the function from the Orlicz space $L_{Q}\left(\mathbb{T}^{2}\right)$ with logarithmic means of rectangular partial sums of double Fourier series, convergent in measure on $\mathbb{T}^{2}$, is of first Baire category in $L_{Q}\left(\mathbb{T}^{2}\right)$.

On the other hand, it is noted, that the regularuty of summation method does not allow to deduce the summability in measure of functional sequence from its convergence in measure (see [1, Remark 1).

In this paper we consider the strong logarithmic means of rectangular partial sums double Fourier series and prove that these means are acting from space $L \log L\left(\mathbb{T}^{2}\right)$ into space $L_{p}\left(\mathbb{T}^{2}\right), 0<p<1$ (see Theorem 3 ). This fact implies the convergence of strong logarithmic means of rectangular partial sums of double Fourier series in measure on $\mathbb{T}^{2}$ to the function $f \in L \log L\left(T^{2}\right)$ (see Corollary 1 ). Uniting these results with statement from [10] we obtain, that the rectangular partial sums of double Fourier series converge in measure for all functions from Orlicz space if and only if their strong Nörlund logarithmic means and strong Nörlund logarithmic means converge in measure (see Theorem [5). Thus, not all classic regular summation methods can improve the convergence in measure of double Fourier series.

The results for summability of logarithmic means of Walsh-Fourier series can be found in [5, 3, 4, 12, 13.

\section{Double Fourier Series and Conjugate Functions}

We denote by $L_{0}=L_{0}\left(\mathbb{T}^{2}\right)$ the Lebesque space of functions that are measurable and finite almost everywhere on $\mathbb{T}^{2}$.

Let $L_{Q}=L_{Q}\left(\mathbb{T}^{2}\right)$ be the Orlicz space [8] generated by Young function $Q$, i.e. $Q$ is a convex continuous even function such that $Q(0)=0$ and

$$
\lim _{u \rightarrow+\infty} \frac{Q(u)}{u}=+\infty, \lim _{u \rightarrow 0} \frac{Q(u)}{u}=0 .
$$

This space is endowed with the norm

$$
\|f\|_{L_{Q}\left(\mathbb{T}^{2}\right)}=\inf \left\{k>0: \iint_{\mathbb{T}^{2}} Q(|f(x, y)| / k) d x d y \leq 1\right\} .
$$

In particular, if $Q(u)=u \log ^{+} u, \log ^{+} u:=1_{\{u>1\}} \log u$, then the corresponding space will be denoted by $L \log L\left(\mathbb{T}^{2}\right)$. 
Given a function $f \in L_{1}\left(\mathbb{T}^{2}\right)$, its double Fourier series is defined by

$$
\sum_{(n, m) \in \mathbb{Z}^{2}} \widehat{f}(m, n) e^{i m x} e^{i n y}
$$

where $\mathbb{Z}:=\{\ldots,-1,0,1,2, \ldots\}$ and

$$
\widehat{f}(m, n)=\frac{1}{4 \pi^{2}} \iint_{\mathbb{T}^{2}} f(x, y) e^{-i m x} e^{-i n y} d x d y
$$

are the Fourier coefficients of the function $f$.

Denote by $S_{n, m}(f ; x, y)$ the $(n, m)$ th symmetric rectangular partial sums of series (1). As is well-known, we have

$$
S_{n, m}(f ; x, y)=\frac{1}{\pi^{2}} \iint_{\mathbb{T}^{2}} f(s, t) D_{n}(x-s) D_{m}(y-t) d s d t
$$

and

$$
D_{n}(u):=\frac{\sin ((n+1 / 2) u)}{2 \sin (u / 2)}
$$

is the Dirichlet kernel.

One can associate three conjugate series to the double Fourier series (1):

$$
\widetilde{f}^{(1,0)} \sim \sum_{(j, k) \in \mathbb{Z}^{2}}(-i \operatorname{sign} j) \widehat{f}(j, k) e^{i(j x+k y)}
$$

(conjugate with respect to the first variable),

$$
\widetilde{f}^{(0,1)} \sim \sum_{(j, k) \in \mathbb{Z}^{2}}(-i \operatorname{sign} k) \widehat{f}(j, k) e^{i(j x+k y)}
$$

(conjugate with respect to the second variable)

$$
\widetilde{f}^{(1,1)} \sim \sum_{(j, k) \in \mathbb{Z}^{2}}(-i \operatorname{sign} j)(-i \operatorname{sign} k) \widehat{f}(j, k) e^{i(j x+k y)}
$$

(conjugate with respect to both variables).

As is well known, if $f$ is an integrable function then

$$
\begin{gathered}
\widetilde{f}^{(1,0)}(x, y)=\mathrm{p} \cdot \mathrm{v} \cdot \frac{1}{\pi} \int_{\mathbb{T}} \frac{f(s, y)}{2 \tan \left(\frac{x-s}{2}\right)} d s, \\
\widetilde{f}^{(0,1)}(x, y)=\mathrm{p} \cdot \mathrm{v} \cdot \frac{1}{\pi} \int_{\mathbb{T}} \frac{f(x, t)}{2 \tan \left(\frac{y-t}{2}\right)} d t
\end{gathered}
$$

and

$$
\widetilde{f}^{(1,1)}(x, y)=\text { p.v. } \frac{1}{\pi^{2}} \iint_{\mathbb{T}^{2}} \frac{f(s, t)}{2 \tan \left(\frac{x-s}{2}\right) 2 \tan \left(\frac{y-t}{2}\right)} d s d t .
$$

Privalov's theorem (see e.g. [16], vol. II, p. 121) immediately implies the a. e. existence of $\widetilde{f}^{(1,0)}$ and $\widetilde{f}^{(0,1)}$ under the assumption $f \in L_{1}\left(\mathbb{T}^{2}\right)$. The a. e. existence of $\widetilde{f}^{(1,1)}$ for $f \in L \log L\left(\mathbb{T}^{2}\right)$ was proved by Zygmund [17, 15. 
We shall consider the symmetric rectangular partial sums of series (13)-(15) defined by

$$
\begin{aligned}
& \widetilde{S}_{n, m}^{10}(f ; x, y):=\sum_{|j| \leq n} \sum_{|k| \leq m}(-i \operatorname{sign} j) \widehat{f}(j, k) e^{i(j x+k y)}, \\
& \widetilde{S}_{n, m}^{01}(f ; x, y):=\sum_{|j| \leq n|k| \leq m} \sum_{\mid}(-i \operatorname{sign} k) \widehat{f}(j, k) e^{i(j x+k y)}
\end{aligned}
$$

and

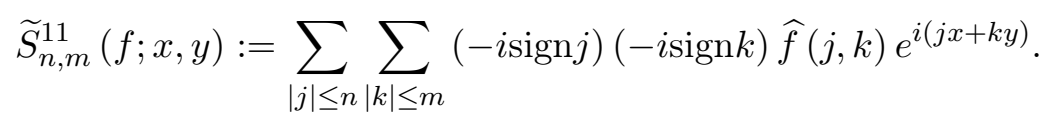

It follows from (2) that

$$
\begin{aligned}
& \widetilde{S}_{n, m}^{10}(f ; x, y)=\frac{1}{\pi^{2}} \iint_{\mathbb{T}^{2}} f(s, t) \widetilde{D}_{n}(x-s) D_{m}(y-t) d s d t \\
& \widetilde{S}_{n, m}^{01}(f ; x, y)=\frac{1}{\pi^{2}} \iint_{\mathbb{T}^{2}} f(s, t) D_{n}(x-s) \widetilde{D}_{m}(y-t) d s d t
\end{aligned}
$$

and

$$
\widetilde{S}_{n, m}^{11}(f ; x, y)=\frac{1}{\pi^{2}} \iint_{\mathbb{T}^{2}} f(s, t) \widetilde{D}_{n}(x-s) \widetilde{D}_{m}(y-t) d s d t
$$

where

$$
\widetilde{D}_{m}(u):=\frac{1}{2 \tan (u / 2)}-\frac{\cos ((m+1) u)}{2 \sin (u / 2)}, m=1,2, \ldots
$$

is the conjugate Dirichlet kernel.

In this paper we also consider the following operators

$$
\begin{aligned}
& \widetilde{\bar{S}}_{n, m}^{10}(f ; x, y)=\frac{1}{\pi^{2}} \iint_{\mathbb{T}^{2}} f(s, t) \widetilde{D}_{n}(x-s) \bar{D}_{m}(y-t) d s d t, \\
& \widetilde{S}_{n, m}^{01}(f ; x, y)=\frac{1}{\pi^{2}} \iint_{\mathbb{T}^{2}} f(s, t) \bar{D}_{n}(x-s) \widetilde{D}_{m}(y-t) d s d t
\end{aligned}
$$

and

$$
\bar{S}_{n, m}(f ; x, y)=\frac{1}{\pi^{2}} \iint_{\mathbb{T}^{2}} f(s, t) \bar{D}_{n}(x-s) \bar{D}_{m}(y-t) d s d t,
$$

where $\bar{D}_{n}(u)$ is a modified Dirichlet kernel defined by

$$
\bar{D}_{n}(u):=\frac{\sin (n u)}{2 \tan (u / 2)} \text {. }
$$




\section{Strong Riesz Logarithmic and Strong Nörlund Logarithmic} MEANS

The strong Riesz logarithmic means, strong Nörlund logarithmic means and strong Fejér means of rectangular partial sums $\widetilde{S}_{i, j}^{a b} f$ defined by

$$
\begin{gathered}
\widetilde{R}_{n, m}^{a b}(f ; x, y):=\frac{1}{l_{n} l_{m}} \sum_{i=0}^{n} \sum_{j=0}^{m} \frac{\left|\widetilde{S}_{i, j}^{a b}(f ; x, y)\right|}{(i+1)(j+1)}, \\
\widetilde{\tau}_{n, m}^{a b}(f ; x, y):=\frac{1}{l_{n} l_{m}} \sum_{i=0}^{n} \sum_{j=0}^{m} \frac{\left|\widetilde{S}_{i, j}^{a b}(f ; x, y)\right|}{(n-i+1)(m-j+1)}, \\
\widetilde{\sigma}_{n, m}^{a b}(f ; x, y):=\frac{1}{(n+1)(m+1)} \sum_{i=0}^{n} \sum_{j=0}^{m}\left|\widetilde{S}_{i, j}^{a b}(f ; x)\right|, a, b=0,1 .
\end{gathered}
$$

Denote

$$
\widetilde{R}_{n, m}^{00}(f)=R_{n, m}(f), \widetilde{S}_{n, m}^{00}(f)=S_{n, m}(f), \widetilde{\tau}_{n, m}^{00}(f)=\tau_{n, m}(f), \widetilde{\sigma}_{n, m}^{00}(f)=\sigma_{n, m}(f) .
$$

In [6], in particular, it is proved that the following estimation is true.

Theorem 2. Let $f \in L \log L\left(\mathbb{T}^{2}\right)$ and $0<p<1$. Then for any $a, b=0,1$ the following estimation holds

$$
\left(\iint_{\mathbb{T}^{2}}\left(\sup _{n, m} \widetilde{\sigma}_{n, m}^{(a, b)}(f ; x, y)\right)^{p} d x d y\right)^{1 / p} \leq c_{1} \iint_{\mathbb{T}}|f(x, y)| \log ^{+}|f(x, y)| d x d y+c_{2} .
$$

Appling Hardy's transformation, we obtain

$$
\begin{aligned}
& l_{n} l_{m} \widetilde{R}_{n, m}^{a b}(f ; x, y) \\
& =\sum_{i=0}^{n-1} \sum_{j=0}^{m-1} \frac{\widetilde{\sigma}_{i, j}^{a b}(f ; x, y)}{(i+2)(j+2)} \\
& +\sum_{j=0}^{m-1} \frac{1}{j+2} \widetilde{\sigma}_{n, j}^{a b}(f ; x, y) \\
& +\sum_{i=0}^{n-1} \frac{1}{i+2} \widetilde{\sigma}_{i, m}^{a b}(f ; x, y) \\
& +\widetilde{\sigma}_{n, m}^{a b}(f ; x, y) .
\end{aligned}
$$


Consequently, from Theorem 2 we obtain

$$
\begin{aligned}
& \left(\iint_{\mathbb{T}^{2}}\left(\widetilde{R}_{n, m}^{a b}(f ; x, y)\right)^{p} d x d y\right)^{1 / p} \\
\leq & 4\left(\iint_{\mathbb{T}^{2}}\left(\sup _{n, m} \widetilde{\sigma}_{n, m}^{(a, b)}(f ; x, y)\right)^{p} d x d y\right)^{1 / p} \\
\leq & c_{1} \iint_{\mathbb{T}^{2}}|f(x, y)| \log ^{+}|f(x, y)| d x d y+c_{2} \quad\left(f \in L \log L\left(\mathbb{T}^{2}\right)\right) .
\end{aligned}
$$

Since

$$
\begin{aligned}
& \left(\int_{\mathbb{T}}\left(\sup _{n} \sigma_{n}(f ; x)\right)^{p} d x\right)^{1 / p},\left(\int_{\mathbb{T}}\left(\sup _{n} \widetilde{\sigma}_{n}(f ; x)\right)^{p} d x\right)^{1 / p} \\
\leq & c_{1} \int_{\mathbb{T}}|f(x)| d x, f \in L_{1}(\mathbb{T}), 0<p<1 .
\end{aligned}
$$

analogously, for one dimensional case we can prove that

$$
\begin{aligned}
& \left(\int_{\mathbb{T}}\left(R_{n}(f ; x)\right)^{p} d x\right)^{1 / p},\left(\int_{\mathbb{T}}\left(\widetilde{R}_{n}(f ; x)\right)^{p} d x\right)^{1 / p} \\
\leq & c_{1} \int_{\mathbb{T}}|f(x)| d x, f \in L_{1}(\mathbb{T}), 0<p<1,
\end{aligned}
$$

where $\sigma_{n}(f ; x), \widetilde{\sigma}_{n}(f ; x), R_{n}(f ; x)$ and $\widetilde{R}_{n}(f ; x)$ are strong Fejér and strong Riesz means of Fourier series and conjugate Fourier series.

\section{Main Results}

Theorem 3. Let $f \in L \log L\left(\mathbb{T}^{2}\right)$ and $0<p<1$. Then the following estimation holds

$$
\left(\iint_{\mathbb{T}^{2}}\left(\tau_{n, m}(f ; x, y)\right)^{p} d x d y\right)^{1 / p} \leq c_{1} \iint_{\mathbb{T}^{2}}|f(x, y)| \log ^{+}|f(x, y)| d x d y+c_{2} .
$$

Theorem 4. Let $f \in L \log L\left(\mathbb{T}^{2}\right)$ and $0<p<1$. Then

$$
\iint_{\mathbb{T}^{2}}\left(\frac{1}{l_{n} l_{m}} \sum_{i=0}^{n} \sum_{j=0}^{m} \frac{\left|S_{i, j}(f ; x, y)-f(x, y)\right|}{(n-i+1)(m-j+1)}\right)^{p} d x d y \rightarrow 0
$$

as $n, m \rightarrow \infty$. 
Corollary 1. Let $f \in L \log L\left(\mathbb{T}^{2}\right)$. Then

$$
\frac{1}{l_{n} l_{m}} \sum_{i=0}^{n} \sum_{j=0}^{m} \frac{\left|S_{i, j}(f ; x, y)-f(x, y)\right|}{(n-i+1)(m-j+1)} \rightarrow 0
$$

in measure on $\mathbb{T}^{2}$, as $n, m \rightarrow \infty$.

Uniting these results with statement 1 of Tkebuchava we obtain.

Theorem 5. The following conditions are equalent

a)

$$
L_{Q}\left(\mathbb{T}^{2}\right) \subset L \log L\left(\mathbb{T}^{2}\right)
$$

b) the strong Nörlund logarithmic means of double Fourier series for all functions from Orlicz space $L_{Q}\left(\mathbb{T}^{2}\right)$ converges in measure on $\mathbb{T}^{2}$;

c) the Nörlund logarithmic means of double Fourier series for all functions from Orlicz space $L_{Q}\left(\mathbb{T}^{2}\right)$ converges in measure on $\mathbb{T}^{2}$;

\section{Proof of Main Results}

Proof of Theorem 3. Set $\alpha_{n}(t):=\sin ((n+1) t), \beta_{n}(t):=\cos ((n+1) t)$. Then we can write

$$
\begin{aligned}
& S_{n-k}(f ; x) \\
= & \frac{1}{\pi} \int_{\mathbb{T}} f(t) \frac{\sin ((n-k+1 / 2)(x-t))}{2 \sin ((x-t) / 2)} d t \\
= & \frac{1}{\pi} \int_{\mathbb{T}} f(t) \sin ((n+1)(x-t)) \frac{\cos ((k+1 / 2)(x-t))}{2 \sin ((x-t) / 2)} d t \\
& -\frac{1}{\pi} \int_{\mathbb{T}} f(t) \cos ((n+1)(x-t)) \frac{\sin ((k+1 / 2)(x-t))}{2 \sin ((x-t) / 2)} d t \\
= & \frac{1}{\pi} \int_{\mathbb{T}} f(t) \sin ((n+1)(x-t)) \\
& \times\left(\frac{\cos ((k+1 / 2)(x-t))}{2 \sin ((x-t) / 2)}-\frac{\cos ((x-t) / 2)}{2 \sin ((x-t) / 2)}\right) d t
\end{aligned}
$$




$$
\begin{aligned}
+ & \frac{1}{\pi} \int_{\mathbb{T}} f(t) \frac{\sin ((n+1)(x-t))}{2 \tan ((x-t) / 2)} d t \\
- & \frac{1}{\pi} \int_{\mathbb{T}} f(t) \cos ((n+1)(x-t)) \frac{\sin ((k+1 / 2)(x-t))}{2 \sin ((x-t) / 2)} d t \\
=- & \frac{\alpha_{n}(x)}{\pi} \int_{\mathbb{T}} f(t) \beta_{n}(t) \widetilde{D}_{k}(x-t) d t \\
+ & \frac{\beta_{n}(x)}{\pi} \int_{\mathbb{T}} f(t) \alpha_{n}(t) \widetilde{D}_{k}(x-t) d t \\
+ & \frac{1}{\pi} \int_{\mathbb{T}} f(t) \frac{\sin ((n+1)(x-t))}{2 \tan ((x-t) / 2)} d t \\
& -\frac{\beta_{n}(x)}{\pi} \int_{\mathbb{T}} f(t) \beta_{n}(t) \frac{\sin ((k+1 / 2)(x-t))}{2 \sin ((x-t) / 2)} d t \\
& -\frac{\alpha_{n}(x)}{\pi} \int_{\mathbb{T}} f(t) \alpha_{n}(t) \frac{\sin ((k+1 / 2)(x-t))}{2 \sin ((x-t) / 2)} d t \\
= & -\alpha_{n}(x) \widetilde{S}_{k}\left(f \beta_{n} ; x\right)+\beta_{n}(x) \widetilde{S}_{k}\left(f \alpha_{n} ; x\right) \\
- & -\beta_{n}(x) S_{k}\left(f \beta_{n} ; x\right)-\alpha_{n}(x) S_{k}\left(f \alpha_{n} ; x\right)+\bar{S}_{n+1}(f ; x)
\end{aligned}
$$

Hence

$$
\begin{aligned}
\tau_{n}(f ; x): & =\frac{1}{l_{n}} \sum_{k=0}^{n} \frac{\left|S_{k}(f ; x)\right|}{n-k+1} \leq \widetilde{R}_{n}\left(f \beta_{n}, x\right)+\widetilde{R}_{n}\left(f \alpha_{n}, x\right) \\
& +R_{n}\left(f \beta_{n}, x\right)+R \sigma_{n}\left(f \alpha_{n}, x\right)+\bar{S}_{n+1}(f ; x) .
\end{aligned}
$$

Since

$$
\left(\int_{\mathbb{T}}\left|S_{n}(f ; x)\right|^{p} d x\right)^{1 / p} \leq c_{p} \int_{\mathbb{T}}|f(x)| d x
$$

from (9) we conclude that

(11)

$$
\left(\int_{\mathbb{T}}\left(\tau_{n}(f ; x)\right)^{p} d x\right)^{1 / p} \leq c_{p} \int_{\mathbb{T}}|f(x)| d x, f \in L_{1}(\mathbb{T}), 0<p<1, f \in L_{1}(\mathbb{T}) .
$$


Now, we consider rectangular partial sums of double Fourier series. From (10) we can write

$$
\begin{aligned}
S_{n-i, m-j}(f ; x, y)= & S_{n-i}\left(S_{m-j}(f ; y) ; x\right) \\
& =-\alpha_{n}(x) \widetilde{S}_{i}\left(S_{m-j}(f ; y) \beta_{n} ; x\right) \\
& +\beta_{n}(x) \widetilde{S}_{i}\left(S_{m-j}(f ; y) \alpha_{n} ; x\right) \\
& -\beta_{n}(x) S_{i}\left(S_{m-j}(f ; y) \beta_{n} ; x\right) \\
& -\alpha_{n}(x) S_{i}\left(S_{m-j}(f ; y) \alpha_{n} ; x\right) \\
& +\bar{S}_{n+1}\left(S_{m-j}(f, y) ; x\right) \\
: & =\sum_{s=1}^{4} I_{s}(i, j ; x, y)+\bar{S}_{n+1}\left(S_{m-j}(f, y) ; x\right) .
\end{aligned}
$$

Now, we turn our attention to $I_{1}(i, j ; x, y)$. From (10) we have

$$
\begin{aligned}
I_{1}(i, j ; x, y)= & -\alpha_{n}(x) S_{m-j}\left(\widetilde{S}_{i}\left(f \beta_{n} ; x\right) ; y\right) \\
= & \alpha_{n}(x) \alpha_{m}(y) \widetilde{S}_{j}\left(\widetilde{S}_{i}\left(f \beta_{n} ; x\right) \beta_{m} ; y\right) \\
& -\alpha_{n}(x) \beta_{m}(y) \widetilde{S}_{j}\left(\widetilde{S}_{i}\left(f \beta_{n} ; x\right) \alpha_{m} ; y\right) \\
& +\alpha_{n}(x) \beta_{m}(y) S_{j}\left(\widetilde{S}_{i}\left(f \beta_{n} ; x\right) \beta_{m} ; y\right) \\
& +\alpha_{n}(x) \alpha_{m}(y) S_{j}\left(\widetilde{S}_{i}\left(f \beta_{n} ; x\right) \alpha_{m} ; y\right) \\
& -\alpha_{n}(x) \bar{S}_{m+1}\left(\widetilde{S}_{i}\left(f \beta_{n} ; x\right) ; y\right) \\
= & \alpha_{n}(x) \alpha_{m}(y) \widetilde{S}_{i j}^{11}\left(f \beta_{n} \beta_{m} ; x, y\right) \\
& -\alpha_{n}(x) \beta_{m}(y) \widetilde{S}_{i j}^{11}\left(f \beta_{n} \alpha_{m} ; x, y\right) \\
& +\alpha_{n}(x) \beta_{m}(y) \widetilde{S}_{i j}^{10}\left(f \beta_{n} \beta_{m} ; x, y\right) \\
& +\alpha_{n}(x) \alpha_{m}(y) \widetilde{S}_{i j}^{10}\left(f \beta_{n} \alpha_{m} ; x, y\right) \\
& -\alpha_{n}(x) \widetilde{\bar{S}}_{i, m+1}\left(f \beta_{n} ; x, y\right) \\
= & \sum_{l=1}^{4} I_{1 l}(i, j ; x, y)+I_{15}(i, m ; x, y) .
\end{aligned}
$$

From (8) we have

$$
\begin{aligned}
& \iint_{\mathbb{T}^{2}}\left(\frac{1}{l_{n} l_{m}} \sum_{i=0}^{n} \sum_{j=0}^{m} \frac{\left|I_{11}(i, j ; x, y)\right|}{(i+1)(j+1)}\right)^{p} d x d y \\
\leq & \iint_{\mathbb{T}^{2}}\left|\widetilde{R}_{n, m}^{11}\left(f \beta_{n} \beta_{m} ; x, y\right)\right|^{p} d x d y
\end{aligned}
$$




$$
\leq c_{1} \iint_{\mathbb{T}^{2}}|f(x, y)| \log ^{+}|f(x, y)| d x d y+c_{2} .
$$

Analogously, we can prove that

$$
\begin{aligned}
& \iint_{\mathbb{T}^{2}}\left(\frac{1}{l_{n} l_{m}} \sum_{i=0}^{n} \sum_{j=0}^{m} \frac{\left|I_{1 l}(i, j ; x, y)\right|}{(i+1)(j+1)}\right)^{p} d x d y \\
\leq & c_{1} \iint_{\mathbb{T}^{2}}|f(x, y)| \log ^{+}|f(x, y)| d x d y+c_{2}, l=2,3,4 .
\end{aligned}
$$

Now, we turn our attention to $I_{15}(i, m ; x, y)$. Since

$$
\begin{gathered}
\widetilde{\bar{S}}_{i, m+1}^{10}\left(f \beta_{n} ; x, y\right)=\widetilde{S}_{i}\left(\bar{S}_{m+1}(f ; y) \beta_{n} ; x\right), \\
f(\cdot, y) \in L \log L(\mathbb{T}), \text { for a.e. } y \in \mathbb{T} \text { and } f \in L \log L\left(\mathbb{T}^{2}\right)
\end{gathered}
$$

and

$$
\int_{\mathbb{T}}\left|\bar{S}_{m+1}(f ; x, y)\right| d x \leq c_{1} \int_{\mathbb{T}}|f(x, y)| \log ^{+}|f(x, y)| d x+c_{2}
$$

from (9) we obtain

$$
\begin{aligned}
& \left.\int_{\mathbb{T}}\left(\frac{1}{l_{n}} \sum_{i=0}^{n} \frac{\left|\widetilde{S}_{i, m+1}^{10}\left(f \beta_{n} ; x, y\right)\right|}{i+1}\right)^{p} d x\right)^{1 / p} \\
\leq & \int_{\mathbb{T}}\left|\bar{S}_{m+1}(f ; x, y)\right| d x \\
\leq & c_{1} \int_{\mathbb{T}}|f(x, y)| \log ^{+}|f(x, y)| d x+c_{2},
\end{aligned}
$$

Consequently,

$$
\begin{aligned}
& \iint_{\mathbb{T}^{2}}\left(\frac{1}{l_{n} l_{m}} \sum_{i=0}^{n} \sum_{j=0}^{m} \frac{\left|I_{15}(i, m ; x, y)\right|}{(i+1)(j+1)}\right)^{p} d x d y \\
\leq & c_{1} \iint_{\mathbb{T}^{2}}|f(x, y)| \log ^{+}|f(x, y)| d x d y+c_{2} .
\end{aligned}
$$

Combining (13)-(16) we get

$$
\begin{aligned}
& \iint_{\mathbb{T}^{2}}\left(\frac{1}{l_{n} l_{m}} \sum_{i=0}^{n} \sum_{j=0}^{m} \frac{\left|I_{1}(i, j ; x, y)\right|}{(i+1)(j+1)}\right)^{p} d x d y \\
\leq & c_{1} \iint_{\mathbb{T}^{2}}|f(x, y)| \log ^{+}|f(x, y)| d x d y+c_{2} .
\end{aligned}
$$


Analogously, we can prove

$$
\begin{aligned}
& \iint_{\mathbb{T}^{2}}\left(\frac{1}{l_{n} l_{m}} \sum_{i=0}^{n} \sum_{j=0}^{m} \frac{\left|I_{s}(i, j ; x, y)\right|}{(i+1)(j+1)}\right)^{p} d x d y \\
\leq & c_{1} \iint_{\mathbb{T}^{2}}|f(x, y)| \log ^{+}|f(x, y)| d x d y+c_{2}, s=2,3,4 \\
& \left(\iint_{\mathbb{T}^{2}}\left(\frac{1}{l_{n} l_{m}} \sum_{i=0}^{n} \sum_{j=0}^{m} \frac{\left|\bar{S}_{n+1}\left(S_{m-j}(f, y) ; x\right)\right|}{i+1}\right)^{p} d x d y\right)^{1 / p} \\
= & \left(\iint_{\mathbb{T}^{2}}\left(\frac{1}{l_{n} l_{m}} \sum_{i=0}^{n} \sum_{j=0}^{m} \frac{\left|S_{m-j}\left(\bar{S}_{n+1}(f, x) ; y\right)\right|}{i+1}\right)^{p} d x d y\right)^{1 / p} \\
= & \left(\iint_{\mathbb{T}^{2}}\left(\frac{1}{l_{m}} \sum_{j=0}^{m} \frac{\left|S_{m-j}\left(\bar{S}_{n+1}(f, x) ; y\right)\right|}{i+1} d x d y\right)^{1 / p}\right. \\
\leq & c_{1} \iint_{\mathbb{T}^{2}}|f(x, y)| \log ^{+}|f(x, y)| d x d y+c_{2} .
\end{aligned}
$$
3.

Combining (12), (17), (18) and (19) we complete the proof of Theorem

By the density of polynomials and by virtue of standard arguments [16] we can see the validity of Theorem 4

\section{REFERENCES}

[1] G. Gát, U. Goginava, G. Tkebuchava, Convergence of logarithmic means of multiple Walsh-Fourier series. Anal. Theory Appl. 21 (2005), no. 4, 326-338.

[2] Gát, G.; Goginava, U.; Tkebuchava, G. Convergence in measure of logarithmic means of quadratical partial sums of double Walsh-Fourier series. J. Math. Anal. Appl. 323 (2006), no. 1, 535-549.

[3] G. Gát, U. Goginava, K. Nagy, On the Marcinkiewicz-Fejér means of double Fourier series with respect to the Walsh-Kaczmarz system. Studia Sci. Math. Hungar. 46 (2009), no. 3, 399-421.

[4] G. Gát and U. Goginava, uniform and $L$-convergence of logarithmic means of WalshFourier series, Acta Math. Sin. (Engl. Ser.) 22 (2006), no. 2, 497-506.

[5] U. Goginava, The weak type inequality for the maximal operator of the Marcinkiewicz-Fejér means of the two-dimensional Walsh-Fourier series. J. Approx. Theory 154 (2008), no. 2, 161-180.

[6] L. Gogoladze, The $(H, k)$-summability of multiple trigonometric Fourier series. (Russian) Izv. Akad. Nauk SSSR Ser. Mat. 41 (1977), no. 4, 937-958.

[7] R. Getsadze, On the divergence in measure of multiple Fourier seties, Some problems of functions theory, 4 (1988), 84-117(in Russian).

[8] M. A. Krasnosel'skii and Ya. B. Rutickii, Convex functions and Orlicz space(English translation), P. Noorhoff (Groningen, 1961). 
[9] S. V. Konyagin, Divergence with respect to measure of multiple Fourier series. (Russian) Mat. Zametki 44 (1988), no. 2, 196-201, 286; translation in Math. Notes 44 (1988), no. 1-2, 589-592 (1989)

[10] G. Tkebuchava, Subsequences of partial sums of multiple Fourier and Fourier-Walsh series. Bull. Georgian Acad. Sci. 169 (2004), no. 2, 252-253.

[11] G. Tkebuchava, Divergence in measure of logarithmic means of multiple Fourier series. Bull. Georgian Acad. Sci. 170 (2004), no. 2, 224-225

[12] O. Szász, On the logarithmic means of rearranged partial sums of Fourier series, Bull. Amer. Math. Soc. 48 (1942), 705-711.

[13] K. Yabuta, Quasi-Tauberian theorems, applied to the summability of Fourier series by Riesz's logarithmic means, Tôhôku Math. Journ. 22 (1970), 117-129.

[14] L. V. Zhizhiashvili, Some problems of multidimensional harmonic analysis, Tbilisi, TGU, 1996 (Russian).

[15] L. V. Zhizhiashvili, Trigonometric Fourier Series and Their Conjugates, Mathematics and its Applications, 372. Kluwer Academic Publishers Group, Dordrecht, 1996.

[16] A. Zygmund, Trigonometric Series, vol. 1, Cambridge Univ. Press, Cambridge, 1959.

[17] A. Zygmund, On the boundary values of functions of several complex variable, Fund. Math. 36 (1949), 207-235.

U. Goginava, Department of Mathematics, Faculty of Exact and Natural Sciences, Iv. Javakhishvili Tbilisi State University, Chavchavadze str. 1, Tbilisi 0128, Georgia

E-mail address: zazagoginava@gmail.com

L. Gogoladze, Department of Mathematics, Faculty of Exact and Natural Sciences, Iv. Javakhishvili Tbilisi State University, Chavchavadze str. 1, Tbilisi 0128, Georgia

E-mail address: lgogoladze1@hotmail.com 